\title{
§ 8 Wachsende Spannungen zwischen den Rechtsordnungen?
}

Die Untersuchung hat bislang gezeigt, dass innerstaatliche Gerichte zu wichtigen Partnern der Menschenrechtsgerichte geworden sind und in zahlreichen Situationen und Konstellationen bereit sind, diese im Rahmen der innerstaatlichen Umsetzung ihrer Entscheidungen zu unterstützen. Dabei hat die Untersuchung ebenfalls deutlich gemacht, dass nationale Gerichte sich in den wenigsten Fällen als simple „Erfüllungsgehilfen“ der Menschenrechtsgerichte verstehen. Vielmehr beharren sie regelmäßig auf einer gewissen Kontrolle über die Entscheidungen und bringen nicht selten zum Ausdruck, dass sie notfalls gar gewillt sind einer Entscheidung die Befolgung zu versagen, wenn sie dies für erforderlich halten.

Beispiele aus jüngerer Zeit legen nahe, dass Gerichte gar verstärkt dazu übergehen Entscheidungen der Menschenrechtsgerichte zu kontrollieren und deren Wirkungen einzuschränken. Die Spannungen zwischen Rechtsordnungen scheinen also zuzunehmen. Auf diese Entwicklung soll im vorliegenden Kapitel eingegangen werden. In einem ersten Schritt soll aufgezeigt werden, wie verschiedene - auch vormals sehr offene und völkerrechtsfreundliche - Gerichte in jüngerer Zeit dazu übergegangen sind, die Entscheidungen der Menschenrechtsgerichte einer verstärkten Kontrolle zu unterziehen (1.). Bei näherem Betrachten zeigt sich allerdings, dass es nur in ganz seltenen Fällen zu eigentlichen Konfrontationen kommt, die eine Verletzung der Befolgungspflicht nach Art. 46 Abs. 1 EMRK bzw. 68 Abs. 1 AMRK nach sich ziehen (2.). In der großen Mehrheit der Fälle schränken Gerichte lediglich die über die eigentliche Bindungswirkung hinausgehenden Wirkungen (res interpretata) ein. Statt um eigentlichen Widerstand handelt es sich also eher um einen Beleg dafür, dass viele Gerichte eine aktivere Rolle im Mehrebenengefüge einfordern. Dies dürfte nicht so sehr als Angriff auf die Menschenrechtsgerichte zu verstehen sein als vielmehr als Reaktion auf deren Erstarken (3.). 


\section{Verstärkte Kontrolle internationaler Judikate}

Zahlreiche Beispiele aus jüngerer Zeit legen nahe, dass sich die Haltung innerstaatlicher Gerichte gegenüber den Menschenrechtsgerichten in einem Wandel befindet. So scheinen Gerichte bestrebt, die Entscheidungen aus Straßburg bzw. San José einer verstärkten Kontrolle zu unterziehen und deren Wirkungen gegebenenfalls einzuschränken. ${ }^{1}$ Insbesondere wird beobachtet, dass sich eine zunehmende Anzahl von Gerichten auf die Verfassung als Schranke des Einbezugs völkerrechtlicher Pflichten im innerstaatlichen Rechtsraum beruft. ${ }^{2}$

Zwar ist es kein neues Phänomen, dass Gerichte regelmäßig auf einer gewissen Letztkontrollbefugnis beharren. Zu denken sei nur an die Positionierung zahlreicher Höchstgerichte gegenüber dem Gerichtshof der Europäischen Union. ${ }^{3}$ Gerade im europäischen Menschenrechtssystem haben viele Gerichte zudem früh erklärt, dass sie auch nicht bereit sind dem EGMR uneingeschränkt zu folgen. Einige sind von vorherein lediglich bereit die Entscheidungen der Menschenrechtsgerichte in eigenen Verfahren zu berücksichtigen, was die Möglichkeit des Abweichens immer bereits impliziert. Dies kommt besonders deutlich in der Rechtsprechung des deutschen Bundesverfassungsgerichts zum Ausdruck. ${ }^{4}$ Der österreichische Verfassungshof hat bereits 1987 in seiner berühmten Entscheidung Miltner klar gemacht, dass trotz des Verfassungsrangs der EMRK in Österreich im Falle eines Konflikts zwischen Verfassung und EMRK in der Auslegung des EGMR erstere den Vorrang genösse. ${ }^{5}$

Trotzdem scheint die Entwicklung in jüngerer Zeit neue Dimensionen anzunehmen. ${ }^{6}$ Sie scheint sich in einen größeren Trend wachsender Kritik insbesondere am

\footnotetext{
${ }^{1}$ Siehe auch Giuseppe Martinico, National courts and judicial disobedience to the ECHR: a comparative overview, in Oddný Mjöll Arnardóttir \& Antoine Buyse (Hrsg.), Shifting Centres of Gravity in Human Righs Protection: Rethinking relations between the ECHR, EU and national legal orders (London/New York: Routledge 2016), 59-78; Marten Breuer (Hrsg.), Principled Resistance to ECtHR Judgments - A New Paradigm? (Berlin/Heidelberg: Springer 2019).

${ }^{2}$ Fulvio Palombino, Compliance with International Judgments: Between Supremacy of International Law and National Fundamental Principles, Zeitschrift für ausländisches öffentliches Recht und Völkerrecht 75 (2015), 503-529; siehe bereits Anne Peters, Supremacy Lost: International Law Meets Domestic Constitutional Law, Vienna Online Journal on International Constitutional Law 3 (2009), 170-198.

${ }^{3}$ Siehe dazu mit Beispielen Anne Peters, The Globalization of State Constitutions, in Janne E. Nijman \& André Nollkaemper (Hrsg.), New Perspectives on the Divide Between National and International Law (Oxford: Oxford University Press 2007), 251-308, 266 f.

${ }^{4}$ Siehe dazu $\S 7$ unter 1.2 .

${ }^{5}$ Verfassungsgerichtshof, B 267/86, Erkenntnis vom 14. Oktober 1987, VfSlg 11.500/1987 = EuGRZ 1988, 166. Siehe dazu Anna Gamper, Austria: Endorsing the Convention System, Endorsing the Constitution, in Patricia Popelier et al. (Hrsg.), Criticism of the European Court of Human Rights. Shifting the Convention System: Counter-Dynamics at the National and EU Level (Cambridge: Intersentia 2016), 75-102, $77 \mathrm{ff}$.

${ }^{6}$ So auch Marten Breuer, ,Principled Resistance' to ECtHR Judgments: Dogmatic Framework and Conceptual Meaning, in Marten Breuer (Hrsg.), Principled Resistance to ECtHR Judgments - A New Paradigm? (Berlin/Heidelberg: Springer 2019), 3-34.
} 
EGMR einzureihen, den viele in einer tiefen Krise sehen. ${ }^{7}$ Immer wieder machen dabei auch Entscheidungen nationaler Gerichte Schlagzeilen, in denen diese die Menschenrechtsgerichte kritisieren oder sich ihnen gar richtiggehend entgegenstellen. Für viel Diskussion gesorgt hat etwa das russische Verfassungsgericht. Dieses entschied 2015 mit Verweis auf andere europäische Verfassungsgerichte wie das deutsche Bundesverfassungsgericht und die italienische Corte Costituzionale, dass es ihm als oberstes Gericht Russlands zustehe, Entscheidungen des EGMR auf ihre Vereinbarkeit mit der russischen Verfassung zu prüfen. ${ }^{8}$ Inzwischen ist die Möglichkeit der systematischen Nachprüfung von EGMR-Entscheidungen durch das Verfassungsgericht explizit gesetzlich geregelt. ${ }^{9}$ Kommt das Verfassungsgericht zum Schluss, dass eine Entscheidung verfassungswidrig ist, führt dies zur Nichtumsetzung der Entscheidung; eine Anpassung der Verfassung schließt das Gesetz kategorisch aus. ${ }^{10}$

Obwohl das Verfassungsgericht betonte, nur in ,extrem seltenen Fällen“ von seinem „Recht auf Widerspruch“ Gebrauch machen zu wollen, ${ }^{11}$ hat es inzwischen bereits zwei EGMR-Entscheidungen für verfassungswidrig erklärt. In einem Urteil vom April 2016 machte es zum ersten Mal von diesem „Vetorecht“ Gebrauch und erklärte die Entscheidung des EGMR in der Sache Anchugov and Gladkov für verfassungswidrig. ${ }^{12}$ In dieser Entscheidung war der EGMR zum Schluss gekommen, dass das in der russischen Verfassung statuierte pauschale Wahlverbot für Häftlinge unverhältnismäßig sei. ${ }^{13}$ Um den Konflikt auf höchster Rechtsebene zu entschärften, verwies der Gerichtshof auch auf die Möglichkeit, die Verfassung konventionskon-

\footnotetext{
${ }^{7}$ Zur Kritik am EGMR siehe umfassend Popelier et al. (Hrsg.), Criticism of the ECtHR.

${ }^{8}$ Verfassungsgericht, Urteil Nr. 21-П/2015 vom 14. Juli 2015, Rn. 4: „Deviation from judgments of the ECtHR, interpreting and applying the Convention, takes place also in the practice of European states, although in exception cases as well and in the presence of sufficiently weighty reasons, including when revealing conventional-constitutional collisions $[\ldots]$. From this perspective the most emblematic is the practice of the Federal Constitutional Court of the Federal Republic of Germany [...].“

${ }^{9}$ Gesetz Nr. 7-KFZ vom 4. Dezember 2015 zur Änderung des Verfassungsgerichtsgesetzes, in Kraft am 15. Dezember 2015, in englischer Übersetzung verfügbar in der Sammlung der Venice Commission, Dokument CDL-REF(2016)006, einsehbar unter http://www.venice.coe.int/webforms/documents/?pdf=CDL-REF(2016)006-e (besucht am 22. September 2019).

${ }^{10}$ Siehe dazu Venice Commission, Final Opinion on the Amendments of the Federal Constitutional Law on the Constitutional Court, CDL-AD(2016)016, Adopted by the Venice Commission at its 107th Plenary Session, Venice, 10-11 June 2016, Rn. 26.

${ }^{11}$ Verfassungsgericht, Urteil Nr. 21-П/2015 (2015), Rn. 6.

${ }^{12}$ Verfassungsgericht, Urteil Nr. 12-П/2016 vom 19. April 2016, offizielle englische Übersetzung verfügbar unter http://www.ksrf.ru/en/Decision/Judgments/Documents/2016_April_19_12-P.pdf (zuletzt besucht am 18. September 2019). Siehe dazu Pietro Pustorino, Russian Constitutional Court and the execution ,à la carte ' of ECtHR judgments, Questions of International Law 32 (2016), 5-18; Ivan Kleimenov, Judgment of the Constitutional Court of the Russian Federation no 12-P/2016: Refusal to execute judgments of ECHR or the search for compromise between Russian and international law?, Questions of International Law 32 (2016), 19-39; Natalia Chaeva, The Russian Constitutional Court and its Actual Control over the ECtHR Judgement in Anchugov and Gladkov, EJIL: Talk!, 26. April 2016, http://www.ejiltalk.org/author/nataliachaeva/.

${ }^{13}$ EGMR, Anchugov and Gladkov v. Russia, Beschwerde-Nrn. 11157/04 und 15162/05, Urteil vom 4. Juli 2013.
} 
form auszulegen. ${ }^{14}$ Das Verfassungsgericht kam jedoch zum Schluss, dass es nicht möglich sei die klare und eindeutige Verfassungsbestimmung anders denn als Verbot auszulegen. In der Folge erklärte es die Straßburger Entscheidung zwar partiell für verfassungswidrig, ließ aber insofern einen kleinen Gesprächsraum offen, als dass es auf die Möglichkeit einer Anpassung durch den Gesetzgeber verwies. ${ }^{15}$

Auch im zweiten Fall befand das Verfassungsgericht das der Prüfung unterzogene Straßburger Urteil für verfassungswidrig. Dabei handelte es sich um das Entschädigungsurteil in der Sache Yukos, in welchem der EGMR den Beschwerdeführern eine rekordhohe Geldsumme zugesprochen hatte. ${ }^{16}$ Bemerkenswerterweise hatte die Venedig-Kommission in ihrer Analyse der Gesetzesrevision, die auf die Verfassungsprüfung der Straßburger Rechtsprechung zielte, bezweifelt, dass die Zahlung einer Entschädigung jemals zu einem Konflikt mit der Verfassung Anlass geben könnte. ${ }^{17}$ Insofern erstaunt es wenig, dass die Entscheidung des russischen Verfassungsgerichts eher konstruiert wirkt. ${ }^{18}$ Das Gericht kam zum Schluss, dass die Entrichtung einer Entschädigungszahlung an die Teilhaber einer Firma, welche Steuerhinterziehung betrieben hatte, gegen die verfassungsmäßigen Prinzipien der Fairness und Gleichheit verstoßen würde. ${ }^{19}$

Während die Spannungen zwischen Straßburg und Moskau angesichts des schwierigen Verhältnisses Russlands zum EMRK-System nicht so sehr erstaunen mögen, ${ }^{20}$ ist bemerkenswert, dass auch Gerichte aus Staaten, die bislang durch ihre offene Haltung gegenüber den Menschenrechtsgerichten auffielen, in jüngerer Zeit einen schärferen Ton anschlagen.

\footnotetext{
${ }^{14}$ Ibid., Rn. 111: ,[...] there may be various approaches to addressing the question of the right of convicted prisoners to vote. In the present case, it is open to the respondent Government to explore all possible ways in that respect and to decide whether their compliance with Article 3 of Protocol No. 1 can be achieved through some form of political process or by interpreting the Russian Constitution by the competent authorities - the Russian Constitutional Court in the first place - in harmony with the Convention in such a way as to coordinate their effects and avoid any conflict between them."

${ }^{15}$ Verfassungsgericht, Urteil Nr. 12-П/2016 (2016), Rn. 5.5.

${ }^{16}$ EGMR, Neftyanaya Kompaniya Yukos v. Russia, Beschwerde-Nr. 14902/04, Urteil (Just Satisfaction) vom 31. Juli 2014.

${ }^{17}$ Venice Commission, Final Opinion on the amendments to the Federal Constitutional Law on the Constitutional (Fn. 10), Rn. 28-30.

${ }^{18}$ Siehe auch Ausra Padskocimaite, Constitutional Courts and (Non)execution of Judgments of the European Court of Human Rights: A Comparison of Cases from Russia and Lithuania, Zeitschrift für ausländisches öffentliches Recht und Völkerrecht 77 (2017), 651-684, 680.

${ }^{19}$ Verfassungsgericht, Urteil Nr. 1-П/2017 vom 19. Januar 2017, offizielle englische Übersetzung verfügbar unter http://www.ksrf.ru/en/Decision/Judgments/Documents/2017_January_19_1-P.pdf (zuletzt besucht am 18. September 2019). Siehe dazu Maxim Timofeyev, Money Makes the Court Go Round: The Russian Constitutional Court's Yukos Judgment, Verfassungsblog, 26. Januar 2017, http://verfassungsblog.de/money-makes-the-court-go-round-the-russian-constitutionalcourts-yukos-judgment/.

${ }^{20}$ Siehe allgemein Lauri Mälksoo \& Wolfgang Benedek (Hrsg.), Russia and the European Court of Human Rights: The Strasbourg Effect (Cambridge: Cambridge University Press 2018).
} 
So hat das oberste argentinische Gericht, bislang einer der wichtigsten Verbündeten des IAGMR und Vorreiter des ,nuevo constitutionalismo“ in Lateinamerika, ${ }^{21}$ jüngst eine Rechtsprechungsänderung vorgenommen. Das Gericht verlangt nun, dass Entscheidungen des IAGMR „Grundprinzipien der argentinischen Rechtsordnung " beachten müssten, um umgesetzt zu werden. Der Gerichtshof hatte zuvor die Aufhebung zweier Urteile verlangt, welche seiner Ansicht nach die Meinungsfreiheit der beiden Journalisten verletzen. ${ }^{22}$ In der Anordnung dieser Maßnahme sah das oberste Gericht zum einen eine Kompetenzüberschreitung von Seiten des IAGMR. ${ }^{23}$ Zum anderen erkannte es darin eine Verletzung der Verfassung. Die Umsetzung des Urteils sei nicht mit Grundprinzipien der argentinischen Rechtsordnung vereinbar (,juridicamente imposible a la luz de los principios fundamentales del derecho publico argentino"). ${ }^{24}$ Denn die Anordnung des IAGMR würde seine Stellung als oberstes Gericht Argentiniens, wie sie in der Verfassung ausdrücklich verankert sei (Art. 108), in Frage stellen und stattdessen den IAGMR an die Spitze der Judikative stellen. ${ }^{25}$ Die Tatsache, dass es selbst das oberste Judikativorgan sei (,órgano supremo y cabeza del Poder Judicial“), gehöre aber zweifellos zu den ,nicht derogierbaren Prinzipien“"(,principios inconmovibles“) nach Art. 27 der Verfassung. ${ }^{26}$ Denn diese Bestimmung behalte einen Souveräntitsbereich der argentinischen Rechtsordung vor, der Völkerrecht nicht weichen könne. ${ }^{27}$ Statt zum Schluss zu kommen, dass es selbst die Anordnung mangels expliziter Gründe, welche die Durchbrechung der Rechtskraft erlauben, nicht umsetzen könne, qualifizierte es die Anordnung als verfassungswidrig.

Ein anderes bemerkenswertes Beispiel ist das italienische Verfassungsgericht. Dieses hatte seit seinen „Zwillingsurteilen“ aus dem Jahr 2007 einen sehr Straßburgfreundlichen Kurs verfolgt; zuweilen wurde ihm gar vorgehalten, zu offen gegenüber dem EGMR zu sein. ${ }^{28}$ In jüngerer Zeit hingegen macht das Gericht insbesondere

\footnotetext{
${ }^{21} \mathrm{Vgl}$. Alexandra Huneeus, Rejecting the Inter-American Court: Judicialization, National Courts, and Regional Human Rights, in Javier Couso et al. (Hrsg.), Cultures of Legality. Judicialization and Political Activism in Latin America (Cambridge: Cambridge University Press 2010), $112-138,113 \mathrm{f}$.

${ }^{22}$ IAGMR, Caso Fontevecchia y D'Amico vs. Argentina, Urteil (Fondo, Reparaciones y Costas) vom 29. November 2011, Tenor Nr. 2: „El Estado debe dejar sin efecto la condena civil impuesta a los señores Jorge Fontevecchia y Hector D'Amico así como todas sus consecuencias, en el plazo de un año contado a partir de la notificación de la presente Sentencia, en los términos del párrafo 105 de la misma."

${ }^{23}$ Siehe dazu $\S 6$ unter 1.

${ }^{24}$ Corte Suprema de Justicia de la Nación, Ministerio de Relaciones Exteriores y Culto s/ informe sentencia dictada en el caso, Fontevecchia y D'Amico vs. Argentina ' por la Corte Interamericana de Derechos Humanos, Urteil vom 14. Februar 2017, 340:47, Rn. 16. Siehe dazu bereits $\S 6$ unter 2 .

${ }^{25}$ Ibid., Rn. 17: „Revocar la sentencia firme dictada por este Tribunal implica privarlo de su character de organo supremo del Poder Judicial argentino y sustituirlo por un tribunal internacional [...].“

${ }^{26}$ Ibid., Rn. 17.

${ }^{27}$ Ibid., Rn. 16.

${ }^{28}$ Siehe dazu $§ 7$ unter 2.2.a).
} 
dadurch von sich reden, dass es sich internationalen Gerichten widersetzt. In seiner zu internationalen Bekanntheit gelangten Entscheidung Nr. 238/2014 erklärte es de facto eine Entscheidung des Internationalen Gerichtshofs für verfassungswidrig. ${ }^{29}$ Später drohte es auch dem Gerichtshof der Europäischen Union seinen Ungehorsam für den Fall an, dass dieser seine Position nicht ändere. Denn das Verfassungsgericht erkannte in einer zuvor ergangenen Entscheidung aus Luxemburg eine Verletzung des Legalitätsprinzips und des Rückwirkungsverbots. ${ }^{30}$

Gegenüber dem EGMR hat der Verfassungshof bislang noch nicht Gebrauch gemacht von der „,nuklearen Option“, eine Entscheidung für verfassungswidrig zu erklären. Trotzdem hat er seinen vormals sehr offenen Kurs korrigiert. Einen ersten Schritt in diese Richtung unternahm er im Folgeurteil zur Entscheidung in der Sache Maggio gegen Italien. ${ }^{31}$ In dieser Entscheidung hielt der Verfassungshof fest, das Interesse an der Umsetzung von Entscheidungen des EGMR müsse gegen andere von der Verfassung geschützte Interessen abgewogen werden, in diesem Fall insbesondere die Prinzipien von Gleichheit und Solidarität. ${ }^{32}$ Im Ergebnis gewichtete das Verfassungsgericht die Interessen an einem ausgeglichenem Finanzhaushalt höher und sah die Einschränkung der in Rede stehenden Grundrechte als gerechtfertigt an. ${ }^{33}$ Die Konsequenz war, dass die in den „Zwillingsentscheidungen“ entwickelte Doktrin ${ }^{34}$ gerade nicht zur Anwendung gelangte und das zugrunde liegende Gesetz damit nicht für verfassungswidrig erklärt wurde. Obwohl der EGMR eine Anpassung der Rechtslage gar nicht verlangt hatte und es damit nicht um die Umsetzung einer angeordneten Maßnahme im engeren Sinne ging, lässt das Gericht der Entscheidung damit nicht die sonst gewährten weitreichenden Wirkungen zukommen.

Noch weiter ausgedehnt hat es die Anforderungen, welche es im Rahmen der Urteilsumsetzung an untere italienische Gerichte stellt, schließlich in einer Entscheidung aus dem Jahr 2015. ${ }^{35}$ Mit dieser Entscheidung schränkt das Gericht die Berücksichtigungspflicht der Rechtsprechung des EGMR durch italienische Gerichte erheblich ein und verlangt eine systematische „Nachprüfung“ von EGMREntscheidungen, bevor diese über Art. 117 der Verfassung einbezogen werden. ${ }^{36}$ Die Entscheidung wird als Abkehr von den „Zwillingsentscheidungen“ qualifiziert und hat zum Ergebnis, dass die Funktion des Mechanismus der Verfassungskontrolle,

\footnotetext{
${ }^{29}$ Corte Costituzionale, Urteil Nr. 238/2014 vom 22. Oktober 2014.

${ }^{30}$ Corte Costituzionale, Verordnung Nr. 24/2017 vom 23. November 2016.

${ }^{31}$ EGMR, Case of Maggio and others v. Italy, Beschwerde-Nrn. 46286/09, 52851/08, 53727/08, 54486/08 und 56001/08, Urteil vom 31. Mai 2011. Siehe dazu bereits $\S 6$ unter 1.

${ }^{32}$ Corte Costituzionale, Urteil Nr. 264/2012 vom 19. November 2011, Rn. 5.3.

${ }^{33} \mathrm{Ibid}$.

${ }^{34}$ Siehe $\S 5$ unter 4.1.c).

${ }^{35}$ Corte Costituzionale, Urteil Nr. 49/2015 vom 26. März 2015.

${ }^{36}$ Auch dabei ging es also nicht um die eigentliche Befolgung einer EGMR-Entscheidung gegen Italien, sondern deren Eignung, als Maßstab der Verfassungskontrolle zu fungieren und damit über den entschiedenen Einzelfall hinaus Wirkung zu erlangen.
} 
um der EMRK in der Auslegung durch Straßburg weitreichende Wirkungen zu verleihen, erheblich relativiert wird. ${ }^{37}$

Die Konsequenz der Entscheidung ist insbesondere, dass das Gericht in Zukunft, abgesehen von Pilot-Urteilen, welche es immer zu beachten gelte, nur noch dazu bereit ist konsolidierter Rechtsprechung aus Straßburg zu folgen. ${ }^{38}$ Für die Frage, wann von „konsolidierter Rechtsprechung“ auszugehen ist, gab das Gericht unteren Gerichten darüber hinaus Kriterien an die Hand. Zum einen machte es nicht-konsolidierte Rechtsprechung an Unstimmigkeiten innerhalb des EGMR fest, die dann zum Ausdruck kommen, wenn abweichende Meinungen vorliegen. Zum anderen deute es auf eine nicht konsolidierte Rechtsprechung hin, wenn eine Entscheidung von der bisherigen Rechtsprechung des EGMR abweicht oder nicht von der Großen Kammer gefällt worden ist. Schließlich hätten Gerichte sich daran zu orientieren, ob die Auslegung durch den EGMR besonders innovativ und neuartig sei. Damit stellt es sich im Ergebnis auch gegen die dynamische Auslegungsmethode des EGMR. ${ }^{39}$

Im Ergebnis kam das Verfassungsgericht in diesem Fall zum Schluss, dass die in Rede stehende Entscheidung des EGMR quasi ein „Ausreißer“ sei. Es verlangt von unteren Gerichten in solchen Fällen nun, ,die einzelne Entscheidung in den Gesamtkontext der Rechtsprechung des Europäischen Gerichtshofs einzuordnen, um daraus einen Sinn abzuleiten, der mit dieser Rechtsprechung vereinbar ist und der unter keinen Umständen gegen die Verfassung verstößt. “40

Obwohl diese Entscheidung nicht per se EMRK-widrig ist, wie sogleich noch ausführlicher darzulegen sein wird, ist sie aus völkerrechtlicher Perspektive dennoch nicht unproblematisch. ${ }^{41}$ Denn sie stellt offen die Autorität des EGMR zur Auslegung der EMRK in Frage, indem das Verfassungsgericht dessen systematische Überprüfung verlangt. ${ }^{42}$ Bemerkenswert dabei ist, dass dieses sich dadurch selbst der Möglichkeit beraubt, mit Straßburg in einen Dialog zu treten, weil bereits untere Gerichte den verlangten Test vornehmen sollen. ${ }^{43}$ Aus völkerrechtlicher

\footnotetext{
${ }^{37}$ Davide Paris \& Karin Oellers-Frahm, Zwei weitere völkerrechts, unfreundliche“ Entscheidungen des italienischen Verfassungsgerichtshofs aus dem Jahr 2015 (Nr. 49 und 50), Europäische Grundrechte-Zeitschrift 43 (2016), 245-252, 249.

${ }^{38}$ Corte Costituzionale, Urteil Nr. 49/2015 (2015), Rn. 7: „It is thus only ,consolidated law“ resulting from the case law of the European Court on which the national courts are required to base their interpretation, whilst there is no obligation to do so in cases involving rulings that do not express a position that has not become final.“

${ }^{39} \mathrm{Zu}$ den Kriterien im Einzelnen siehe ibid., Rn. 7.

${ }^{40}$ Ibid., Rn. 6.1. Übersetzung von der Verfasserin.

${ }^{41}$ Paris und Oellers-Frahm qualifizieren sie als völkerrechtsunfreundlich. Paris \& Oellers-Frahm, Zwei weitere völkerrechts,,unfreundliche" Entscheidungen, EuGRZ.

${ }^{42}$ Andrea Pin, A Jurisprudence to Handle with Care: The European Court of Human Rights' Unsettled Case Law, its Authority, and its Future, According to the Italian Constitutional Court, Int'l J. Const. L. Blog, 30. April 2015, http://www.iconnectblog.com/2015/04/mini-symposium-on-ccjudgment-49-2015.

${ }^{43}$ Kritisch dazu Paris \& Oellers-Frahm, Zwei weitere völkerrechts,,unfreundliche“ Entscheidungen, $E u G R Z, 249 \mathrm{f}$.
} 
Perspektive erschiene es allein aus Gründen der Übersichtlichkeit vorzugswürdiger, wenn der EGMR einen Ansprechpartner hätte. Auf diese Punkte wird erneut zurückzukommen sein.

In eine ähnliche Richtung geht schließlich nun auch das Bundesverfassungsgericht, das in einer Entscheidung jüngst die aus der EGMR-Rechtsprechung im weiteren Sinne fließenden Wirkungen - die sog. „Orientierungswirkung "“44 - für deutsche Gerichte weiter konkretisiert und im Ergebnis eingeschränkt hat. In Fällen, in denen es nicht um die Durchsetzung von nach Art. 46 Abs. 1 EMRK für Deutschland verbindlichen Entscheidungen geht - im vorliegenden Fall war das Gericht mit gegen die Türkei ergangenen Entscheidungen konfrontiert - verlangt das Gericht nun eine stärkere „Kontextualisierung“ der Entscheidung..$^{45}$ Nicht nur „ein Moment der Vergleichbarkeit" sei Voraussetzung für die Anwendung einer Rechtsprechung, sondern auch der „rechtskulturelle Hintergrund“ sowie „,spezifische Besonderheiten der deutschen Rechtsordnung, die einer undifferenzierten Übertragung im Sinne einer bloßen ,Begriffsparallelisierung “" entgegenstünden. ${ }^{46}$ Im Ergebnis führt dies wohl dazu, dass die „Orientierungswirkung“ künftig im Wesentlichen auf gegen Deutschland ergangene Entscheidungen beschränkt wird. Denn diese sei da „besonders groß, wo sie sich auf Parallelfälle im Geltungsbereich derselben Rechtsordnung “ beziehe ${ }^{47}$ Darüber hinaus sind es nach dieser Rechtsprechung nur mehr noch „Aussagen zu den Grundwertungen der Konvention“, die zu berücksichtigen sind. ${ }^{48}$

\section{Nichtbefolgung und die Pflichten nach Art. 46 EMRK/68 AMRK}

Diese Beispiele zeigen, dass verschiedene vormals sehr offene Gerichte ihre Haltung gegenüber den Menschenrechtsgerichten in jüngerer Zeit angepasst und bis zu einem gewissen Grad eingeschränkt haben. Die Spannungen zwischen den Rechtsordnungen scheinen insgesamt also zuzunehmen. Dabei ist allerdings wichtig hervorzuheben, dass es nur in den wenigsten Fällen zu eigentlichen Konfrontationen im Sinne echter Rechtskonflikte kommt. Anders ausgedrückt: Nur in seltenen Fällen führt die Weigerung eines nationalen Gerichts dem EGMR bzw. IAGMR zu folgen zu einer Verletzung der Befolgungspflicht nach Art. 46 Abs. 1 EMRK bzw. Art. 68 Abs. 1 AMRK.

Dies liegt zum einen daran, dass Gerichte den Menschenrechtsgerichten wie gesehen weit über die eigentliche Befolgungspflicht hinaus folgen. Viele Gerichte

\footnotetext{
${ }^{44}$ Siehe dazu $\S 5$ unter 1.2. sowie $\S 7$ unter 1.2.

${ }^{45}$ Bundesverfassungsgericht, 2 BvR 1738/12 (Beamtenstreikrecht), Urteil des Zweiten Senats vom 12. Juni 2018, B VerfGE 148, 296, Rn. 132.

${ }^{46}$ Ibid.

${ }^{47}$ Ibid.

${ }^{48}$ Ibid.
} 
beziehen heute die gesamte Rechtsprechung der Menschenrechtsgerichte ein. ${ }^{49}$ Nicht selten nutzen sie gegen andere Staaten ergangene Entscheidungen zudem als Gelegenheit, um allfällige Meinungsunterschiede vorzutragen und ihre internationalen Pendants möglicherweise von ,ihrer“ Lösung zu überzeugen. Dies ist nicht zuletzt Zeugnis gelebter Subsidiarität: Gerichte behalten sich vor, auf dem Weg des gerichtlichen Dialogs Widerspruch anzumelden und so zur Weiterentwicklung der Konventionsgarantien beizutragen. ${ }^{50}$ Dies brachte das italienische Verfassungsgericht jüngst ganz explizit zum Ausdruck: Die Bestimmung des Inhalts der EMRKRechte sei eine gemeinsame Aufgabe von nationalen Gerichten und dem EGMR. ${ }^{51}$ In diesem Sinne seien nationale Gerichte nicht lediglich ,,passive Empfänger eines Auslegungskommandos, das an anderer Stelle in Form eines Gerichtsurteils erteilt wird“.52 Eine ,hierarchische Auferlegung einer bestimmten Interpretation“ lehnt es zumindest da ab, wo noch keine etablierte Rechtsprechung besteht. ${ }^{53}$ Das Verfassungsgericht verwies denn auch ausdrücklich auf drei vor der Großen Kammer anhängige Beschwerden und bringt damit zum Ausdruck, dass es auf ein Einlenken von Seiten Straßburgs hofft. ${ }^{54}$

Aber sogar eine Weigerung eines Gerichts, eine im engeren Sinne bindende Entscheidung durchzusetzen, muss nicht zu einer Verletzung der Befolgungspflicht führen. Dies liegt daran, dass neben Gerichten regelmäßig weitere Akteure an der innerstaatlichen Umsetzung von Entscheidungen beteiligt sind.$^{55}$ Insofern kann eine Entscheidung dem EGMR bzw. IAGMR nicht zu folgen schlicht auch als Einladung an die Legislative oder Exekutive zu verstehen sein, die in ihre Zuständigkeit fallenden Schritte zu unternehmen.

Problematisch sind die (seltenen) Fälle, in denen Gerichte eine internationale Entscheidung für im innerstaatlichen Recht überhaupt nicht vollstreckbar oder verfassungswidrig erklären, denn dies kann bedeuten, dass eine Umsetzung auch durch andere innerstaatliche Akteure nicht zulässig und damit vollkommen ausgeschlossen ist. Dies wird zuweilen als ,,principled resistance“ bezeichnet. ${ }^{56}$ Besonders deutlich wird dies im Falle des russischen Verfassungsgerichts, welches inzwischen

\footnotetext{
${ }^{49}$ Siehe dazu $§ 5$ unter 1.; zur unterschiedlichen Behandlung von res iudicata und res interpretata siehe $\S 7$ unter 2.2.a).

${ }^{50}$ Martinico, National courts and judicial disobedience to the ECHR: a comparative overview, in Mjöll Arnardóttir \& Buyse (Hrsg.), 60. Siehe dazu mit Beispielen aus Frankreich, England und Deutschland Eirik Bjorge, Domestic Application of the ECHR. Courts as Faithful Trustees (Oxford: Oxford University Press 2015), 223 ff.

${ }^{51}$ Corte Costituzionale, Urteil Nr. 49/2015 (2015), Rn. 7.

${ }^{52} \mathrm{Ibid}$. Übersetzung von der Verfasserin.

${ }^{53}$ Ibid. Übersetzung von der Verfasserin.

${ }^{54}$ Ibid., Rn. 6. Siehe dazu auch Martinico, National courts and judicial disobedience to the ECHR: a comparative overview, in Mjöll Arnardóttir \& Buyse (Hrsg.), 76 f., der dieses Beispiel eines „Akts des Ungehorsams“ differenziert betrachtet. Im Ergebnis schränkt das Verfassungsgericht mit dieser Entscheidung jedoch die Möglichkeit eines Dialogs mit Straßburg ein. Siehe dazu Paris \& Oellers-Frahm, Zwei weitere völkerrechts ,unfreundliche“ Entscheidungen, EuGRZ, 250.

${ }^{55}$ Siehe dazu $§ 1$.

${ }^{56}$ Breuer, Dogmatic Framework, in Breuer (Hrsg.).
} 
bereits zwei Entscheidungen des EGMR für nicht mit der russischen Verfassung vereinbar und deshalb nicht umsetzbar erklärt hat. Denn das Gesetz, in dem die Verfassungskontrolle der Straßburger Entscheidungen nun explizit geregelt ist, schließt eine Anpassung der Verfassung aus. ${ }^{57} \mathrm{Zu}$ solchen Ergebnissen kann es auch in Fällen kommen, in denen Gerichte einzelne Entscheidungen mit fundamentalen Prinzipien der eigenen Rechtsordnung oder der „Verfassungsidentität“ unvereinbar erklären, wie es in einer jüngeren Entscheidung etwa das oberste argentinische Gericht getan hat ${ }^{58}$ Denn auch in diesen Fällen kann eine rechtliche Anpassung schwierig oder gar unmöglich sein. ${ }^{59}$

Solche Entscheidungen erinnern an das Prozedere der Anerkennung und Vollstreckung ausländischer Entscheidungen, in dem gemeinhin gewisse Vorbehalte der Rechtsordnung gelten, in der die Entscheidung vollzogen werden soll. Diese werden regelmäßig einer inhaltlichen Kontrolle unterzogen, bevor ihnen das sog. „Exequatur" erteilt wird. Nach schweizerischem Recht etwa kann die Anerkennung dann verweigert werden, wenn eine Entscheidung ,mit dem schweizerischen ordre public offensichtlich unvereinbar" ist ${ }^{60}$ eine ähnliche Ausnahme sieht auch das sog. „Lugano-Übereinkommen“ vor. ${ }^{61}$ Obwohl die Fragestellung eine grundsätzlich andere ist, wenn es um internationale Entscheidungen geht, ${ }^{62}$ steht dahinter letztlich die gleiche (dualistische) Logik: Innerstaatliche Gerichte sind zwar zur Rezeption von Judikaten aus einer anderen Rechtsordnung bereit, aber nur zu den Bedingungen, welche die eigene Rechtsordnung festlegt und über die sie die Kontrolle beanspruchen.

$\mathrm{Zu}$ einer Verletzung der Befolgungspflicht nach den Menschenrechtskonventionen kann es in der Folge kommen, da aus völkerrechtlicher Perspektive diese Einwände bekanntlich nicht zählen - innerstaatliches Recht kann, unabhängig von dessen Rang und Wichtigkeit in der nationalen Rechtsordnung, nicht vorgebracht werden, um die Nichterfüllung völkerrechtlicher Pflichten zu rechtfertigen. ${ }^{63}$ Die

\footnotetext{
${ }^{57}$ Siehe zum Ganzen oben unter 1.

${ }^{58}$ Corte Suprema de Justicia de la Nación, Fontevecchia y D'Amico (2017). In diesem Fall ist das argentinische Gericht schlussendlich aber zu einem Kompromiss gelangt. Siehe Corte Suprema de Justicia de la Nación, Resolution Nr. 4015/17 vom 5. Dezember 2017.

${ }^{59} \mathrm{Zu}$ einem ähnlichen Ergebnis kommt Breuer. Siehe Breuer, Dogmatic Framework, in Breuer (Hrsg.), 14.

${ }^{60}$ Art. 27 Abs. 1 des Bundesgesetzes über das Internationale Privatrecht (IPRG) vom 18. Dezember 1987, SR 291.

${ }^{61}$ Siehe Art. 34 Abs. 1 des Übereinkommens über die gerichtliche Zuständigkeit und die Anerkennung und Vollstreckung von Entscheidungen in Zivil- und Handelssachen vom 30. Oktober 2007, SR 0.275.12.

${ }^{62}$ So schon Jörg Polakiewicz, Die Verpflichtungen der Staaten aus den Urteilen des Europäischen Gerichtshofs für Menschenrechte (Berlin/Heidelberg: Springer 1993), $211 \mathrm{f}$.

${ }^{63} \mathrm{Vgl}$. Art. 3 der Articles on Responsibility of States for Internationally Wrongful Acts, Text adopted by the International Law Commission at its fifty-third session in 2001 (UN Doc. A/56/10); vgl. auch Art. 27 der Wiener Konvention über das Recht der Verträge vom 23. Mai 1969, UNTS Vol. 1155 p. 331. Siehe für eine Diskussion der Anwendbarkeit von Art. 46 WVK in diesem Zusammenhang Breuer, Dogmatic Framework, in Breuer (Hrsg.), 8 f.
} 
Menschenrechtsgerichte ihrerseits verlangen auch im Falle eines Konflikts mit der Verfassung bzw. der Verfassungspraxis, dass ihre Entscheidungen gutgläubig befolgt werden und sich die Staaten folglich anpassen, auch wenn solche Fälle eher selten sind. ${ }^{64}$ In diesem Sinne ist die Berufung auf die Verfassung bzw. einen ,Verfassungskern" keine völkerrechtliche Kategorie und damit kein Grund, der die völkerrechtliche Verantwortlichkeit eines Staates auszuschließen vermöchte. Die Entwicklung eines entsprechenden Ausnahmetatbestandes scheint angesichts der damit verbundenen Gefahren auch nicht wünschenswert. ${ }^{65}$

Allerdings ließe sich überlegen, ob in Einzelfällen davon ausgegangen werden könnte, dass Entscheidungen von EGMR und IAGMR die Schwelle der Nichtigkeit erreichen, womit innerstaatliche Gerichte allenfalls einen Grund hätten, diese nicht $\mathrm{zu}$ beachten. So könnte man argumentieren, dass Kompetenzüberschreitungen durch die Menschenrechtsgerichte oder krass falsche Entscheidungen zur Nichtigkeit der jeweiligen Judikate führen könnten. ${ }^{66}$

Obwohl die Existenz von Nichtigkeitsgründen für internationale Judikate an sich nicht umstritten ist, ist im Einzelnen unklar, wie die Annullierung tatsächlich vollzogen werden soll. Denn die Anerkennung von Nichtigkeitsgründen steht in einem inhärenten Spannungsverhältnis zum Bestehen endgültiger internationaler Entscheidungen und birgt das Risiko, die Autorität des in Rede stehenden internationalen Gerichts zu untergraben. ${ }^{67}$ Entschärft ist die Problematik da, wo systeminterne Berufungsinstanzen bestehen und die Gründe vorgetragen werden können. Während Nichtigkeitsgründe im Zusammenhang mit EGMR-Entscheidungen damit kaum eine Rolle spielen, weil Entscheidungen an die Große Kammer weitergezogen und noch einmal überprüft werden können (Art. 43 EMRK) ${ }^{68}$ besteht diese Möglichkeit im interamerikanischen System nicht. Gerade die heiklen strafrechtlichen Fälle des IAGMR illustrieren, dass ein Korrektiv durchaus erforderlich erscheint. Eine systeminterne Revisionsinstanz schiene dabei das beste Mittel, um Mängel zu rügen und wäre somit auch für das interamerikanische System wünschenswert. So lange eine Art „Große Kammer“ innerhalb des IAGMR aber nicht besteht, bleibt die Frage relevant, wann innerstaatliche Gerichte als Korrektiv

\footnotetext{
${ }^{64}$ Siehe Venice Commission, Final Opinion on the Amendments of the Federal Constitutional Law on the Constitutional Court (Fn. 10), Rn. 24, mit Verweis auf Beispiele von Verfassungsreformen im Zuge von EGMR-Entscheidungen in Griechenland, Ungarn, Italien, der Slovakei und der Türkei. In Lateinamerika hat Chile seine Verfassung aufgrund des IAGMR-Urteils in der Sache „The Last Temptation of Christ" angepasst. Siehe IAGMR, Case of ,,The Last Temptation of Christ" (Olmedo-Bustos et al.) v. Chile, Monitoring Compliance with Judgment, Anordnung vom 28. November 2003.

${ }^{65}$ So auch Palombino, Compliance with International Judgments, ZaöRV, 523.

${ }^{66}$ Siehe zu Nichtigkeitsgründen Oscar Schachter, The Enforcement of International Judicial and Arbitral Decisions, American Journal of International Law 54 (1960), 1-24, 3; siehe ausführlich Bernhard Plamper, Die Nichtigkeit völkerrechtlicher Gerichtsentscheidungen (Frankfurt am Main u. a.: Peter Lang 2010), 19; $127 \mathrm{ff}$.

${ }^{67}$ Karin Oellers-Frahm, Judicial and Arbitral Decisions, Validity and Nullity, Max Planck Encyclopedia of Public International Law, Rn. 3.

${ }^{68}$ Plamper, Die Nichtigkeit völkerrechtlicher Gerichtsentscheidungen, 19; 90 f.
} 
einspringen können und sollen. Auf diese Frage wird im letzten Teil dieser Studie zurückzukommen sein.

Klar ist, dass angesichts der damit verbundenen Gefahren die Anforderungen an die Nichtigkeit sehr hoch sein müssen und es sich nur um absolute Ausnahmefälle handeln kann, soll nicht die Verbindlichkeit internationaler Streitbeilegung in Frage gestellt werden. Die Frage der Nichtigkeit dürfte sich für innerstaatliche Gerichte in der Praxis damit kaum je als Rechtfertigung für die Nichtbefolgung einer Entscheidung eignen.

\section{Fazit: wenig Konfrontation, aber verstärkte Kontrolle}

Fälle, in denen sich Gerichte einem internationalen Judikat entgegenstellen mit dem Ergebnis, dass dieses nicht umgesetzt wird, können also grundsätzlich nicht unter Berufung auf völkerrechtliche Rechtfertigungsgründe gerechtfertigt werden. Obwohl es wichtig ist zu betonen, dass diese Fälle nach wie vor selten sind, ist nicht zu verkennen, dass sie einen Angriff auf die Autorität des internationalen Gerichts bedeuten. ${ }^{69}$ Gemäß dem IAGMR bedroht die Nichtbefolgung von Entscheidungen ,the raison d'être for the functioning " internationaler Gerichte, ${ }^{70}$ und der EGMR kam zum Schluss, dass „,der bewusste Versuch, die Umsetzung eines endgültigen und vollstreckbaren Urteils zu verhindern“ geeignet sei, ,die Glaubwürdigkeit und Autorität der Justiz zu untergraben und ihre Wirksamkeit zu gefährden. " ${ }^{\circ 11}$ Beide Gerichte gehen darüber hinaus davon aus, dass die effektive Befolgung ihrer Urteile ein integraler Teil des Rechts, von einem Gericht gehört zu werden, ist. ${ }^{72}$ Auch wenn nicht davon auszugehen ist, dass vereinzelte solche Angriffe einer internationalen Institution nachhaltigen Schaden zufügen bzw. gar deren Untergang besiegeln, so ist nicht zu verkennen, dass sie sich trotzdem auf die in Rede stehende Institution auswirken und diese zu Anpassungen zwingen können. ${ }^{73}$ So lässt sich etwa beim EGMR in jüngerer Zeit ein stärkerer Rekurs auf Techniken beobachten, die es ihm erlauben mehr Zu-

\footnotetext{
${ }^{69}$ Alexandra Huneeus, Compliance with Judgments and Decisions, in Cesare Romano et al. (Hrsg.), The Oxford Handbook of International Adjudication (Oxford: Oxford University Press 2014), 437-463, 442; Breuer, Dogmatic Framework, in Breuer (Hrsg.), 19.

${ }^{70}$ IAGMR, Case of Baena Ricardo v. Panama, Judgment (Competence), 28. November 2003, Rn. 72.

${ }^{71}$ EMGR, Broniowski v. Poland, Beschwerde-Nr. 31443/96, Urteil (GK) vom 22. Juni 2004, Rn. 175. Siehe auch EGMR, The Authority of the Judiciary, Background Paper zum Seminar Dialogue between judges 2018, verfügbar unter https://www.echr.coe.int/Documents/Seminar_background_paper_2018_ENG.pdf (zuletzt besucht am 22. September 2019), Rn. 6: „Shortcomings in the enforcement of judicial decisions may undermine judicial authority. "Übersetzung von der Verfasserin.

${ }^{72}$ Siehe dazu $§ 1$ unter 1.3 .

${ }^{73}$ Mikael Rask Madsen, Resistance to the European Court of Human Rights: The Institutional and Sociological Consequences of Principled Resistance, in Marten Breuer (Hrsg.), Principled Resistance to ECtHR Judgments - A New Paradigm? (Berlin/Heidelberg: Springer 2019), 35-52, 52.
} 
rückhaltung zu üben. Wendet er diese einseitig zu Gunsten seiner Kritiker an, worauf Untersuchungen hindeuten, wird dies zu einem Problem für seine Glaubwürdigkeit. ${ }^{74}$

Aus diesen Gründen sollte gerichtlicher Widerstand im engeren, oben beschriebenen Sinne klar vom Instrument des gerichtlichen Dialoges unterschieden werden. Zwar gibt es einige Parallelen zwischen den beiden Figuren, und beide können letztlich ganz ähnliche Funktionen erfüllen. Der gerichtliche Dialog dient oft gerade dazu, Meinungsverschiedenheiten auszutragen. Er erlaubt es nationalen Gerichten, unterschiedliche Sichtweisen zu artikulieren und so die Konventionsauslegung mitzubestimmen. Er ist für nationale Gerichte bis zu einem gewissen Grad ein „Recht $\mathrm{zu}$ widersprechen “75 und bildet in diesem Sinne ,, a constructive way for channelling substantive disagreement or criticism “ ${ }^{76}$ Wenn Gerichte den Menschenrechtsgerichten in bindenden Fällen widersprechen, dürften sie vielfach ein ganz ähnliches Ziel verfolgen: Nicht selten dürfte es darum gehen, ihr Gegenüber zum Einlenken zu bewegen und eine Änderung der Rechtslage zu erzwingen. So hat das italienische Verfassungsgericht in seiner Entscheidung, mit welcher es die Umsetzung einer IGH-Entscheidung blockiert hat, ${ }^{77}$ ganz explizit zum Ausdruck gebracht, dass es dadurch ,zu einer wünschenswerten - und von vielen gewünschten - Entwicklung des Völkerrechts" beizutragen hoffe. ${ }^{78}$ Bereits die Androhung von Widerstand kann schließlich dazu dienen, dass sich innerstaatliche Gerichte überhaupt Gehör verschaffen. Widerstand kann so überhaupt erst die Grundlage für einen echten Dialog schaffen, indem es Gerichten dadurch gelingt, eine „gleichberechtigte Konversation zu erzwingen “. ${ }^{79}$ Wenn in Widerstand gegenüber internationalen Gerichten zwar eine gewisse Gefahr für die Autorität internationaler Gerichte und die Stabilität der internationalen Ordnung liegt, mehren sich in jüngerer Zeit denn auch die Stimmen in der Literatur, die darin auch Chancen erkennen und argumentieren, dass dieser letztlich positive Rückwirkungen auf das Völkerrecht haben und längerfristig gar zu dessen Stärkung beitragen könnte. ${ }^{80}$

\footnotetext{
${ }^{74}$ Zum Ganzen siehe Başak Çali, Coping with Crisis: Whither the Variable Geometry in the Jurisprudence the European Court of Human Rights, Wisconsin International Law Journal 35 (2018), 237-276; Mikael Rask Madsen, Rebalancing European Human Rights: Has the Brighton Declaration Engendered a New Deal on Human Rights in Europe?, Journal of International Dispute Settlement 9 (2018), 199-222.

${ }^{75}$ Siehe dazu $§ 2$ unter 1.3 .

${ }^{76}$ Sarah Lambrecht, Assessing the Existence of Criticism of the European Court of Human Rights, in Patricia Popelier et al. (Hrsg.), Criticism of the European Court of Human Rights. Shifting the Convention System: Counter-Dynamics and the National and EU Level (Cambridge: Intersentia 2016), 505-553, 549.

${ }^{77} \mathrm{IGH}$, Jurisdictional Immunities of the State (Germany v. Italy: Greece Intervening), Urteil vom 3. Februar 2012, I.C.J. Reports 2012, 99.

${ }^{78}$ Corte Costituzionale, Urteil Nr. 238/2014 (2014), Conclusions on points of law, Rn. 3.3. Übersetzung von der Verfasserin.

${ }^{79}$ Anne Peters, Rechtsordnungen und Konstitutionalisierung: Zur Neubestimmung der Verhältnisse, Zeitschrift für öffentliches Recht 65 (2010), 3-63, 52; Krisch, Beyond Constitutionalism, $78 \mathrm{ff}$.

${ }^{80}$ Siehe dazu ausführlicher $\S 10$.
} 
Trotzdem spricht vieles dafür, den Begriff des gerichtlichen Dialoges auf Fälle zu beschränken, in denen Gerichte über die Auslegung in künftigen Fällen streiten. Hat ein Menschenrechtsgericht in einem Einzelfall entschieden, ist das „Gespräch“ erst einmal beendet. Dann geht es nicht mehr um die abstrakte Konventionsauslegung, sondern die Durchsetzung einer Entscheidung in einem konkreten Fall. Widerspricht ein innerstaatliches Gericht seinem Gegenüber in dieser Situation mit der Folge, dass eine Entscheidung innerstaatlich nicht umgesetzt werden kann, geht es eher darum diese erneut zu überprüfen und deren Wirkungen zu verhindern. Zwar kann auch dies eine wichtige Funktion erfüllen, etwa im interamerikanischen System, das wie gesehen keine interne Revisionsinstanz und damit keine Möglichkeit kennt, Entscheidungen von einer höheren Instanz zu kontrollieren. Darauf wird später zurückzukommen sein. Trotzdem ist Widerspruch im oben beschriebenen Sinne, nämlich dann, wenn er die innerstaatliche Umsetzung einer Entscheidung gänzlich unmöglich macht, widerrechtlich. In der Literatur wurde vorgeschlagen, für diese Konstellation den Begriff ,dialectic review“ statt gerichtlichen Dialog zu verwenden. ${ }^{81}$ Um die Grenzen zwischen dem ,legalen“ und sogar konventionsrechtlich gewünschten ${ }^{82}$ Dialogieren und den - seltenen - Fällen konventionsrechtlich heiklen Widerspruchs nicht zu verwischen, scheint diese Terminologie in der Tat vorzugswürdig.

Insgesamt ist das Bild bei näherem Betrachten aber wie gesehen weniger dramatisch, als es auf den ersten Blick scheint. Zwar zeigen die untersuchten Beispiele eindeutig, dass auch vormals offene und menschenrechtsfreundliche Gerichte dazu übergegangen sind, die Entscheidungen der Menschenrechtsgerichte einer verstärkten Kontrolle zu unterziehen und deren Umsetzung vom Einhalten gewisser Bedingungen des nationalen Rechts abhängig zu machen. Dies spricht für eine gewisse Re-Nationalisierung der Selbstwahrnehmung von Gerichten. Trotzdem ist damit kein „Kampf der Gerichte“ ausgebrochen, und es scheint zu kurz gegriffen, die Entwicklung als Sieg des Nationalismus und nationaler Interessen über Multilateralismus und das Ende internationaler Gerichtsbarkeit zu lesen. ${ }^{83}$

Plausibler scheint es, die gewandelte Haltung zahlreicher Gerichte auch auf die tatsächlich geänderten Umstände zurückzuführen. Nicht nur ist es heute, auch dank der aktiveren Rolle von Individuen, welche die tatsächliche Umsetzung von zu ihren Gunsten ergangenen Entscheidungen einfordern, schlicht zu einer Intensivierung der Interaktion zwischen den verschiedenen Rechtsordnungen gekommen. Statt als Versuch der Schwächung der Menschenrechtsgerichte könnte die Entwick-

\footnotetext{
${ }^{81}$ Vgl. Robert B. Ahdieh, Between Dialogue and Decreee: International Review of National Courts, New York University Law Review 79 (2004), 2029-2163.

${ }^{82}$ Siehe dazu Teil I.

${ }^{83}$ Siehe auch Madsen et al., die für eine stärkere Differenzierung verschiedener Fälle der „Gegenreaktionen" gegen das Völkerrecht plädieren: Mikael Rask Madsen et al., Backlash against international courts: explaining the forms and patterns of resistance to international courts, International Journal of Law in Context 14 (2018), 197-220.
} 
lung also auch als Reaktion auf deren Erstarken und die enorme Zunahme internationaler Entscheidungen gelesen werden. ${ }^{84}$ Als Konsequenz können sich gerade obere innerstaatliche Gerichte durch mächtige internationale Gerichte „bedroht" sehen und versuchen ihre Stellung zu verteidigen. Martinico etwa bezeichnet dies als den „Preis des Erfolges“ des EGMR. ${ }^{85}$ Bifulco und Paris argumentieren, dass es dem italienischen Verfassungsgericht mit seinen Zeichen, die es nach Straßburg sendet, auch darum gehe, sicherzustellen, dass die Aufgabe italienischer Gerichte nicht auf diejenige eines „Exekutivorgans“ für Entscheidungen des EGMR reduziert wird. ${ }^{86}$

Erkenntnisse aus der politikwissenschaftlichen Forschung legen ferner nahe, dass wachsende institutionelle Macht mit wachsender Politisierung, d. h. öffentlicher Aufmerksamkeit und Debatte sowie auch Widerstand, einhergeht. ${ }^{87}$ Dies stimmt mit der allgemeinen Beobachtung überein, wonach ein „reactive turn“ stattfindet und sich innerstaatliche Gerichte verstärkt gegen die Umsetzung völkerrechtliche Verpflichtungen stellen, was der zunehmenden völkerrechtlichen Regelungsdichte und -intensität und dem dadurch erhöhten Konfliktpotenzial angelastet wird. ${ }^{88}$ Insofern erstaunt nicht, dass gerade internationale Entscheidungen, die mit ihren konkreten Anordnungen deutlich größeres Konfliktpotenzial zu innerstaatlichem Recht als das oft vage generell-abstrakte Völkerrecht bergen, auch auf Ablehnung stoßen ${ }^{89}$ Dies gilt besonders für die Menschenrechtsgerichte, die beide - in unterschiedlichem Maße - durch die Konkretisierung ihrer Entscheidungen dazu beigetragen haben, dass ihre Entscheidungen auf der innerstaatlichen Ebene stärker greifen und leichter umsetzbar werden. ${ }^{90}$ Wie gesehen kann die Durchsetzung der Urteile der Menschenrechtsgerichte innerstaatliche Gerichte in ein richtiggehendes Dilemma führen; so können sie sich gehalten sehen zwischen der Befolgung internationaler Vorgaben und dem Respekt innerstaatlichen Rechts entscheiden zu müs-

\footnotetext{
${ }^{84}$ So auch Nico Krisch, The Backlash against International Courts, Völkerrechtsblog, 16. Dezember 2014, https://voelkerrechtsblog.org/the-backlash-against-international-courts/; ders., Pluralism in International Law and Beyond, in Jean d'Aspremont \& Sahib Singh (Hrsg.), Concenpts for International Law. Contributions to Disciplinary Thought (Cheltenham/Northampton: Edward Elgar 2019), 691-706.

${ }^{85}$ Martinico, National courts and judicial disobedience to the ECHR: a comparative overview, in Mjöll Arnardóttir \& Buyse (Hrsg.), 68.

${ }^{86}$ Bifulco \& Paris, Italienischer Verfassungsgerichtshof, in von Bogdandy et al. (Hrsg.), Rn. 174.

${ }^{87}$ Krisch, Backlash, Völkerrechtsblog, 16. Dezember 2014. Dazu grundlegend Michael Zürn \& Matthias Ecker-Ehrhardt (Hrsg.), Die Politisierung der Weltpolitik. Umkämpfte internationale Institutionen (Berlin: Suhrkamp 2013).

${ }^{88}$ International Law Association (ILA), Study Group on the Engagement of Domestic Courts with International Law, Mapping the Engagement of Domestic Courts with International Law, Final Report by Antonios Tzanakopoulos, Co-Rapporteur of the Study Group, verfügbar unter http:// www.ila-hq.org/index.php/study-groups (besucht am 20. Juli 2017), Rn. 22.

${ }^{89}$ So auch Krisch, Pluralism, in d'Aspremont \& Singh (Hrsg.).

${ }^{90}$ Siehe dazu Teil I.
} 
sen. Die stärkere Involvierung innerstaatlicher Gerichte in der Umsetzung internationaler Entscheidungen führt damit nicht nur zu einer Stärkung; ihre Rolle wird damit auch deutlich komplexer. Damit wird die Frage immer drängender, welche Rolle innerstaatliche Gerichte an der Schnittstelle zwischen Rechtsordnungen wahrnehmen sollen. Mit dieser normativen Frage wird sich das letzte Kapitel der vorliegenden Untersuchung auseinandersetzen.

Open Access Dieses Kapitel wird unter der Creative Commons Namensnennung 4.0 International Lizenz (http://creativecommons.org/licenses/by/4.0/deed.de) veröffentlicht, welche die Nutzung, Vervielfältigung, Bearbeitung, Verbreitung und Wiedergabe in jeglichem Medium und Format erlaubt, sofern Sie den/die ursprünglichen Autor(en) und die Quelle ordnungsgemäß nennen, einen Link zur Creative Commons Lizenz beifügen und angeben, ob Änderungen vorgenommen wurden.

Die in diesem Kapitel enthaltenen Bilder und sonstiges Drittmaterial unterliegen ebenfalls der genannten Creative Commons Lizenz, sofern sich aus der Abbildungslegende nichts anderes ergibt. Sofern das betreffende Material nicht unter der genannten Creative Commons Lizenz steht und die betreffende Handlung nicht nach gesetzlichen Vorschriften erlaubt ist, ist für die oben aufgeführten Weiterverwendungen des Materials die Einwilligung des jeweiligen Rechteinhabers einzuholen. 